\title{
SECULAR EVOLUTIONARY TRENDS IN ELLIPTICALS AND BULGES DUE TO MERGERS
}

\author{
TAPAN K. CHATTERJEE \\ Facultad de Ciencias, Fisico-Matematicas, Universidad A. Puebla, (A.P.1316), Puebla, \\ Mexico
}

Using basically the impulsive approximation and a modification of the method used by Alladin S.M., (1965, Ap.J., 141, 768), and described in detail in Chatterjee T.K., (1990, IAU Col., 124, 519, 569) we study the evolution of binary interacting galaxies, leading ultimately to mergers. Each collision is characterised by the initial separation between the galaxies and the relative velocity therein. In each case the orbital evolution and largescale structural changes in the galaxies are studied by taking into account the change in relative velocity due to dynamical friction, leading ultimately to mergers. The evolution is considered from a time when the gravitational interaction between the progenitor pairs is physically significant (Chatterjee, 1992, Astroph. Sp.Sc., in press).

Results indicate that the expected frequency of merging galaxies, not considering the galaxies to be embedded in massive halos, is $10^{-2} \%$, under favourable environmental conditions. This value falls short of the observational value in the present epoch by an order of magnitude and falls short of the extrapolated past value by two orders of magnitudes. But in median environmental conditions, let alone unfavourable ones, the merger rates are drastically low (Chatterjee, 1992,a). The frequency of different types of progenitor pairs in merger is of the same order of magnitude. The majority of the mergers are achieved in several (2 to 3 ) orbital periods, and only about $10 \%$ of them are achieved in a single orbital period; and only about $1 \%$ of them are due to central impacts. During binary evolution substancial changes in the structures of the participating galaxies take place due to intense tidal effects. Several bursts of star formation will also take place during this time and the resultant dissipation is likely to cause a substancial change in the mass to luminosity ratio $(M / L)$ of the system.

Binary evolution will have its reflection on the fundamental parameter plane for ellipticals (e.g. Kormendy and Djorgovski, 1989, Ann. Rev. Astron. Astr., 27, 235). The variation of the power coefficients of the fundamental plane variables from the pure virial coefficients are indicative of the dependence of the factor of proportionality in the fundamental plane solutions, upon the fundamental plane variables. This factor depends not only upon the formation but also upon the evolutionary history of galaxies. This implies that the structural changes and dissipation undergone during binary evolution and mergers is relevant in defining the finescale structure of the fundamental plane; in particular a curvature is expected to be induced. These secular evolutionary trends may also be applicable to bulges of spirals, as they seem to occupy almost the same fundamental plane as ellipticals.

I would like to thank all those with whom I had helpful discussions, especially Prof. A. Poveda. 


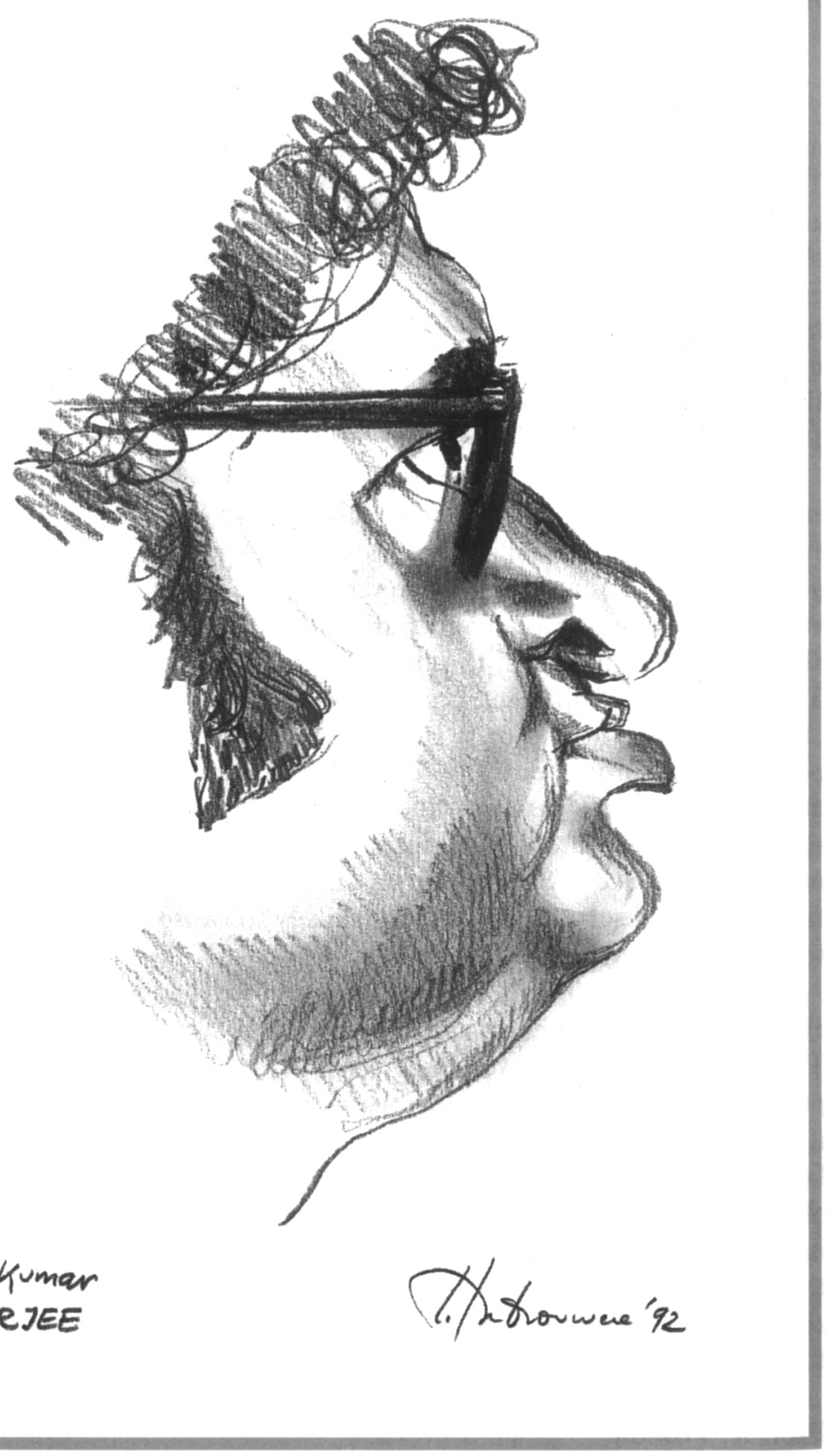

